\title{
Depressed with cancer can respond to antidepressants, but further research is needed to confirm and expand on these findings
}

\author{
Tatsuo Akechi, ${ }^{1}$ Toshi A Furukawa ${ }^{2}$
}

${ }^{1}$ Department of Psychiatry and Cognitive-Behavioral Medicine, Nagoya City University, Nagoya, Japan; ${ }^{2}$ Kyoto University, Kyoto, Japan

Correspondence to Dr Tatsuo Akechi, takechi@med.nagoya-cu.ac.jp

\section{WHAT IS ALREADY KNOWN ON THIS TOPIC?}

The quality of life of patients with cancer is affected not only by their physical illness but also by comorbid psychological conditions, such as depression. Mitchell and colleagues reported that major depression has a point prevalence of $10-20 \%$ in patients with cancer, irrespective of cancer stage. ${ }^{1}$ This prevalence is similar to that seen in patients with other chronic medical illnesses. Both psychological and pharmacological approaches are suggested to be effective for patients with cancer with elevated depressive symptoms. ${ }^{2}$

\section{WHAT THIS PAPER ADDS}

- There is very limited evidence to guide the treatment of patients with cancer with a diagnosis of major depression.

- In particular, there is very little evidence demonstrating the effectiveness of psychological treatments.

- Some evidence exists that antidepressants, alone or in combination with a psychological treatment, may be effective.

\section{LIMITATIONS}

- The identified studies were too heterogeneous with regard to both the participants and the type of treatments to allow meta-analytical synthesis. The authors were therefore unable to provide informative data for clinicians, including number needed to treat or harm.

- Although the present study found several potential biases, including poor allocation concealment (selection bias), unblinded data collection (detection bias) and unspecified primary outcomes (reporting bias) commonly observed in the previous studies, the authors did not provide any clear recommendations for future clinical trials.

\section{WHAT NEXT IN RESEARCH?}

- Even though there is some evidence that patients with cancer with major depression may respond to antidepressants in general, more data are needed regarding the most commonly prescribed antidepressants (ie, SSRIS and SNRIs), because physicians tend to treat patients with cancer as they treat other depressed patients without comorbidity. Comparative efficacy of antidepressants in the treatment of less severe depression (often occurring in association with advanced disease) are unknown.
- Given that patients suffering from clinical depression generally prefer psychological treatments to pharmacological interventions, more studies are needed to investigate the effectiveness of psychological therapies for major depression in patients with cancer. ${ }^{3}$

- In particular, studies of psychological treatments for mild depression in patients with cancer are needed because most depression observed in patients with cancer is generally mild.

\section{DO THESE RESULTS CHANGE YOUR PRACTICES AND WHY?}

Since the present study mainly demonstrated the lack of high-quality clinical trials for major depression among patients with cancer, the findings would not influence our current practice. However, it is useful to note that application of unwanted (but received) interventions has been uniquely associated with poor psychosocial adjustment even though the intervention is psychological treatment, ${ }^{4}$ and that we do not know the potential harms of pharmacological treatments especially for patients with cancer with aggressive anticancer treatments and at advanced stages, as shown by the present study. Clinicians should manage major depression among patients with cancer carefully, as efficacy and harms of commonly used treatments remain largely unknown.

Competing interests TA has received speaking fees and/or research funds from Astra-Zeneca, GlaxoSmithKline, Meiji, MSD, Otsuka, Pfizer, Yoshitomi, Shionogi and NHK enterprise. He has received royalties from Igaku-Shoin and Nanzando. TAF has received lecture fees from Eli Lilly, Meiji, Mochida, MSD, Pfizer and Tanabe-Mitsubishi and consultancy fees from Sekisui and Takeda Science Foundation. He is diplomate of the Academy of Cognitive Therapy and Deputy Editor of the journal EBMH.

doi:10.1136/eb-2014-101923

\section{REFERENCES}

1. Mitchell AJ, Chan M, Bhatti H, et al. Prevalence of depression, anxiety, and adjustment disorder in oncological, haematological, and palliative-care settings: a meta-analysis of 94 interview-based studies. Lancet Oncol 2011;12:160-74.

2. Hart SL, Hoyt MA, Diefenbach $M$, et al. Meta-analysis of efficacy of interventions for elevated depressive symptoms in adults diagnosed with cancer. J Natl Cancer Inst 2012;104:990-1004.

3. McHugh RK, Whitton SW, Peckham AD, et al. Patient preference for psychological vs pharmacologic treatment of psychiatric disorders: a meta-analytic review. J Clin Psychiatry 2013;74:595-602.

4. Reynolds JS, Perrin NA. Mismatches in social support and psychosocial adjustment to breast cancer. Health Psychol 2004;23:425-30.

ABSTRACT FROM: Walker J, Sawhney A, Hansen CH, et al. Treatment of depression in adults with cancer: a systematic review of randomized controlled trials. Psychol Med 2014;44:897-907.

Data sources MEDLINE, EMBASE, PsychINFO and the Cochrane Central Register of Controlled Trials (CENTRAL) from inception to 2012.

Study type included RCTs evaluating the efficacy of treatments for depression in adults with a definite cancer diagnosis and a diagnosis of major depression (according to DSM-IV or International Classification of Diseases-10, ICD-10).

Intervention Any treatment for depression.

Comparison Placebo, standard care or another treatment for depression.

OUTCOMES

Study characteristics Seven RCTs were identified, including 713 participants. Trials often had unclear risk of bias, and meta-analysis was not possible due to heterogeneity.
Results In one trial of women with breast or gynaecological cancer $(n=73)$, mianserin was more effective than placebo in two studies, one lasting 28 days (standardised mean difference (SMD) $=0.58,95 \% \mathrm{CI} 0.13$ to 1.03 ) and other one 42 days ( $\mathrm{SMD}=0.80,0.26$ to 1.34 ). Another trial in women with breast cancer $(n=179)$ found no significant difference between amitriptyline and paroxetine at 8 weeks.

No difference between behavioural activation and problem-solving therapy at up to 12-month follow-up in a trial in women with breast cancer $(n=80)$.

Antidepressants treatment combined with education and problem-solving therapy $(n=200)$ was more effective than usual care, $(S M D=0.43,95 \%$ CI 0.16 to 0.71$)$. 